\title{
A Simple In Vivo Atrial Fibrillation Model of Rat Induced by Transesophageal Atrial Burst Pacing
}

\author{
Atsushi Sugiyama ${ }^{1, *}$, Akira Takahara ${ }^{1}$, Sachiko Honsho ${ }^{1}$, Yuji Nakamura ${ }^{1}$, and Keitaro Hashimoto ${ }^{1}$ \\ ${ }^{1}$ Department of Pharmacology, Interdisciplinary Graduate School of Medicine and Engineering, University of Yamanashi, \\ Tamaho-cho, Nakakoma-gun, Yamanashi 409-3898, Japan
}

Received January 24, 2005; Accepted May 2, 2005

\begin{abstract}
A simple in vivo closed-chest atrial fibrillation (Af) model of rats was developed. Af was reproducibly induced by transesophageal atrial burst pacing for $30 \mathrm{~s}$ in each of the pentobarbital-anesthetized rats, whereas the cardiohemodynamic condition as well as the inducibility and duration of Af episode was stable over time. Moreover, the anti-Af effect of the class Ic drug pilsicainide was confirmed in this model, which was essentially the same as those reported previously in other Af animal models and clinical practice. Thus, this new model may become an alternative to current techniques.
\end{abstract}

Keywords: atrial fibrillation, pilsicainide, pentobarbital

While many experimental models of atrial fibrillation (Af) have been developed, most of them utilize large animals and/or pharmacological interventions $(1,2)$. The former is often expensive, time-consuming, and lacks versatility, whereas the latter may be confounded by drug-drug interactions. Many attempts have been made to improve them, and recently Haugan et al. developed a new Af model of rats using rapid transesophageal atrial pacing during brief episodes of asphyxia (3). In an effort to further improve upon the model, this report describes a more simple and reliable paroxysmal Af model of rats, in which anesthesia was kept with pentobarbital instead of a neuroleptic anesthetic combination consisting of fentanyl citrate, fluanisone, and midazolam (3), thus making the asphyxia dispensable to Af induction. This is a critical new finding and is a significant improvement over the previous rat Af model in which Af was hardly induced in the absence of asphyxia (3). In this report, we describe this new method, which can be completed within $10 \mathrm{~min}$ after the induction of anesthesia. Moreover, in order to test the model, we selected a pure sodium channel blocker pilsicainide, since in Japan, the drug has been most widely used against clinical paroxysmal Af attack $(4,5)$.

Animals were obtained through the Animal Labo-

*Corresponding author. FAX: +81-55-273-6739

E-mail: atsushis@yamanashi.ac.jp ratory for Research of the University of Yamanashi. All experiments were performed in accordance with Guidelines for Animal Experiments of the University of Yamanashi. Male Sprague-Dawley rats weighing $300-$ $400 \mathrm{~g}$ were anesthetized by intra-peritoneal injection of pentobarbital sodium $(50 \mathrm{mg} / \mathrm{kg})$. The trachea was cannulated for artificial respiration (SN-480-7; Shinano, Tokyo). Tidal volume and respiratory rate were set at $10 \mathrm{ml} / \mathrm{kg}$ and 60 strokes/min, respectively. ECG lead II together with the blood pressure at the femoral artery was continuously monitored using a polygraph system (RM-6200; Nihon Kohden, Tokyo) and analyzed using a real-time fully automatic data analysis system (MPVAS/Win; Physio-Tech, Tokyo).

A clinically available 6-French quad-polar electrodes catheter, whose diameter is similar to that of the gastric tube for rats, was inserted into the esophagus under the monitoring of an esophageal electrogram and positioned at a site the lowest threshold could capture the atrium. The pacing pulse used for the induction of Af was rectangular in shape, of $60 \mathrm{~V}$ (about 1.5 times of the diastolic threshold voltage) and 6-ms width. The atrium was paced at a cycle length of $12 \mathrm{~ms}(83 \mathrm{~Hz})$ for $30 \mathrm{~s}$ (=burst pacing) via the distal electrodes pair of the catheter using an electrical stimulator (SEN-7203, Nihon Kohden) and an isolator (SS-201J, Nihon Kohden). Extensive preliminary studies have confirmed that this experimental protocol could be optimal for 
constantly inducing Af in all animals (3). In this study, Af was defined as a period of rapid irregular atrial rhythm with irregular R-R interval lasting at least $2 \mathrm{~s}(6)$. When Af was induced by the burst pacing, its duration was measured. After the assessment of the basal control state, the animals were randomly assigned to two groups; namely, pilsicainide- or saline-administered groups. Then, the therapeutic dose of $1 \mathrm{mg} / \mathrm{kg}$ of pilsicainide $(n=6)$ or a vehicle saline $(n=6)$ was administered in a volume of $1 \mathrm{ml} / \mathrm{kg}$ through the femoral vein $(4,5)$. Effects on the PR interval, QRS width, and QT interval of the ECG, heart rate, mean blood pressure, and Af duration were assessed 10, 20, 30 , and 60 min after the drug administration.

Pilsicainide hydrochloride (Sunrhythm ${ }^{\mathrm{TM}}$ Inj; Daiichi Pharmaceutical, Tokyo) and pentobarbital sodium (Tokyo-Kasei, Tokyo) were purchased. The data are presented as the mean \pm S.E.M. The statistical comparisons of mean values within a group were carried out using one-way repeated-measures analysis of variance (ANOVA) followed by Contrast for statistical analysis between basal values and others, whereas those between the groups were assessed using the unpaired $t$-test. A $P$ value $<0.05$ was considered significant.

Typical tracings of the ECG, esophageal electrogram, and blood pressure during the sinus rhythm are depicted in Fig. 1A, and the time courses of their changes prior to each burst pacing episode are summarized in Fig. 1B. The heart rate, mean blood pressure, PR interval, QRS width, and QT interval at the pre-drug control in the pilsicainide-treated group were $396 \pm 9$ beats $/ \mathrm{min}$, $135 \pm 5 \mathrm{mmHg}, 42 \pm 2 \mathrm{~ms}, 21 \pm 1 \mathrm{~ms}$, and $72 \pm 2 \mathrm{~ms}$, respectively $(n=6)$, while those in the saline-treated group were $430 \pm 16$ beats/min, $138 \pm 4 \mathrm{mmHg}, 41 \pm 3$ $\mathrm{ms}, 21 \pm 1 \mathrm{~ms}$, and $65 \pm 2 \mathrm{~ms}$, respectively $(\mathrm{n}=6)$. No significant difference was detected in the respective pre-drug control values between the groups. In the pilsicainide-treated group, the heart rate and mean blood pressure decreased transiently at $10 \mathrm{~min}$, while no significant change was detected in the ECG parameters. Meanwhile in the saline-treated group, no significant change was detected in any of the parameters during the observation period, indicating the stability of the cardiohemodynamic condition in this new model.

Af was induced in all animals of each group at the pre-drug control. Typical tracings of the ECG showing the burst pacing-induced Af are depicted in Fig. 2A, and the time courses of changes in the Af duration are summarized in Fig. 2B. The Af duration at the pre-drug control in the pilsicainide-treated group was $11.7 \pm 3.5 \mathrm{~s}$ $(\mathrm{n}=6)$, while that in the saline-treated group was $9.1 \pm 3.1 \mathrm{~s}(\mathrm{n}=6)$. No significant difference was detected in the pre-drug control values between the groups. In
A

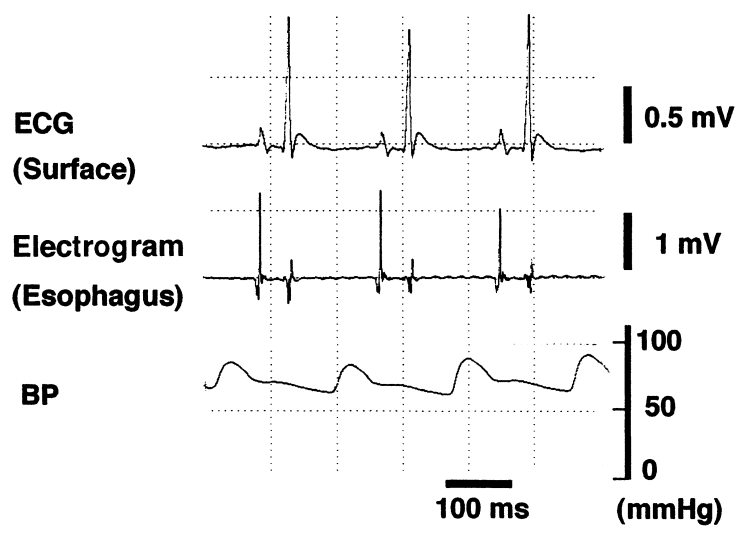

B

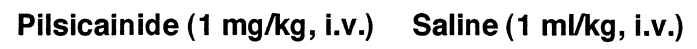

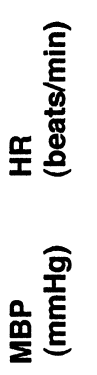
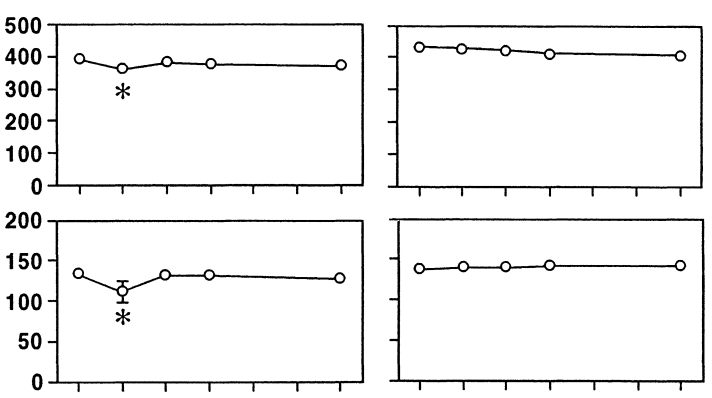

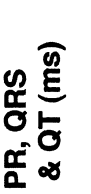
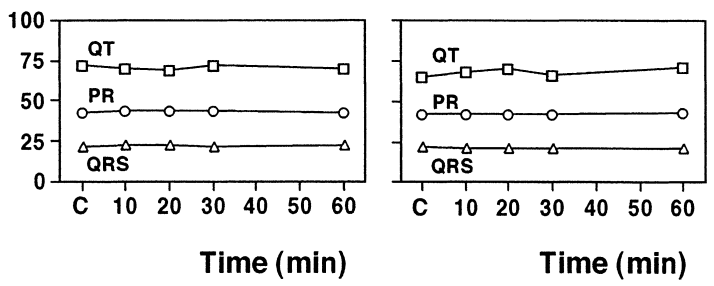

Fig. 1. Cardiovascular effects of pilsicainide $(n=6)$ and saline $(n=6)$. A: Typical tracings of the ECG, esophageal electrogram, and blood pressure before the administration of the drug. B: The time courses of the changes in the heart rate, mean blood pressure, and ECG parameters. ECG: electrocardiogram, BP: blood pressure, HR: heart rate, and MBP: mean blood pressure. ${ }^{*} P<0.05$ vs pre-drug control (C).

the pilsicainide-treated group, the Af duration was significantly shortened to $<35 \%$ of the control value at $10 \mathrm{~min}$, and a similar trend was confirmed at other time points, which is essentially the same as those reported in other Af animal models and clinical practice $(4,5)$, indicating that this new method will be sensitive enough to measure anti-Af effects of such a group of drugs. Meanwhile in the saline-treated group, no significant change was detected in the Af duration or inducibility during the observation period, indicating the reproducibility of the Af episode in this model.

In vivo animal models of Af have been generally considered to be restricted to larger animals, including dogs, sheep, and goats, because of the lack of a critical mass for re-entry, which has prevented their widespread 
A

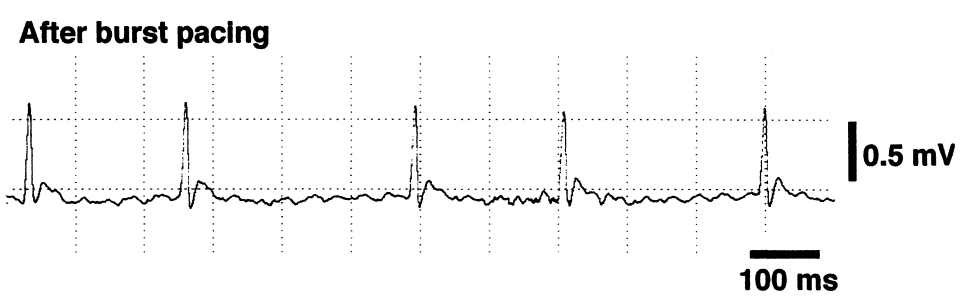

Termination of Af

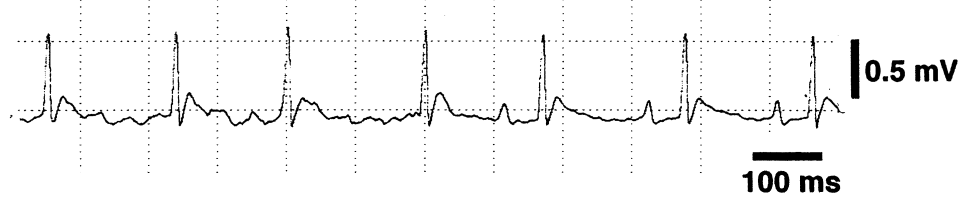

B
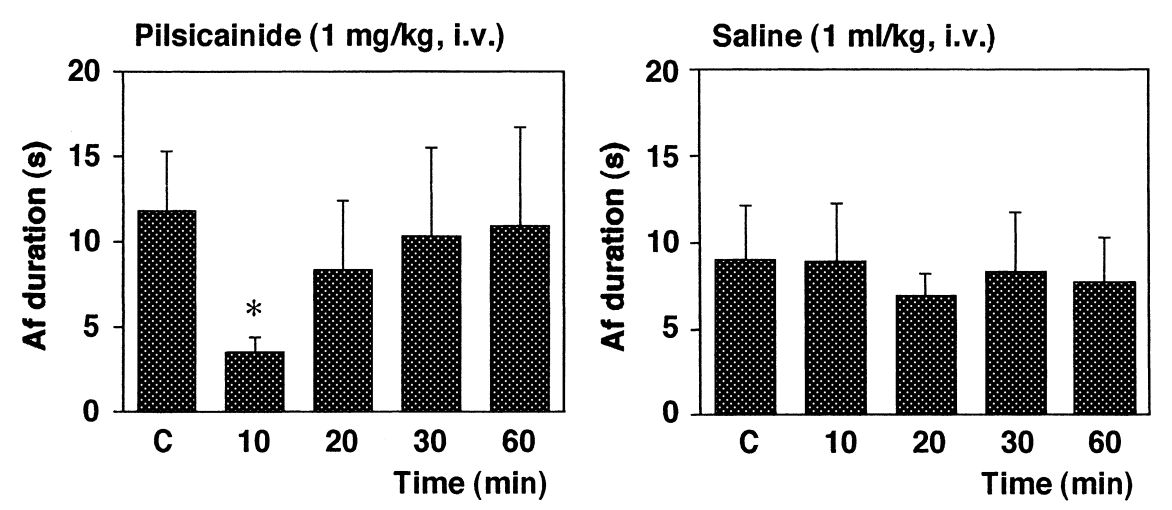

Fig. 2. Anti-Af effects of pilsicainide $(n=6)$ and saline $(n=6)$. (A) Typical tracings of the ECG showing the burst pacinginduced Af before the administration of the drug. The ventricular rate is highly irregular. The QRS complex is narrow and there is no $\mathrm{P}$ wave during Af episode. (B) The time courses of the changes in the Af duration. Af: atrial fibrillation. ${ }^{*} P<0.05 v s$ pre-drug control (C).

application $(1,2)$. Recent studies have demonstrated that Af can be induced in small animals like rats and mice by atrial burst stimulation $(1-3,6)$. However, in the mouse model, induction of Af depends on the cholinergic stimulation (2) or selective atrial fibrosis by overexpression of TGF- $\beta 1$ (6). On the other hand, the rat model needs asphyxia during the atrial burst pacing to increase Af inducibility from $6 \%$ to $91 \%$ (3). In this study, Af inducibility was $100 \%$ without using cholinergic stimulation, asphyxia, or surgical treatment. Therefore, our new method may offer a number of potential advantages to current techniques in terms of feasibility, rapidity, stability, reliability, and economy in testing anti-Af effects of drugs.

In the rat Af model by Haugan et al. (3), the neurolept anesthetic combination was employed for the maintenance of anesthesia, whereas we adopted pentobarbital. There are several reports describing the effects of these anesthetic agents on the cardiovascular system. Briefly,
Skolleborg et al. (7) reported that peripheral circulation was better and that serum corticosterone level was lower in the midazolam/fentanyl/fluanisone anesthesia than in pentobarbital in the rat. Using neonatal rats, Griffioen et al. (8) demonstrated that fentanyl can act on $\mu$-opioid receptors on cardiac vagal neurons and their preceding neurons to reduce GABAergic neurotransmission, resulting in bradycardia via an increase of parasympathetic activity. On the other hand, Lindmar et al. (9) and Chiba and Tsuboi (10) showed that pentobarbital inhibits the acetylcholine release from the postganglionic parasympathetic nerve terminals and/or the ganglionic transmission, resulting in the antivagal effect on the heart. In addition, pentobarbital has been shown to suppress sodium, calcium, and potassium channels at clinically relevant concentrations $(11-13)$, whereas no information was available for any of the neurolept anesthetic combinations on cardiac ion channels. Furthermore, pentobarbital as well as fentanyl was 
reported to exert antiarrhythmic effects on the animal model of ventricular arrhythmias $(14,15)$. After all, this previous knowledge cannot fully explain why pentobarbital anesthesia promotes the onset of Af in rats in this study.

There are some limitations in this study. While the 6French catheter is an effective way to induce Af, feasible to handle, and durable for multiple use, its large surface area along with relatively high direct current may have injured the left atrial posterior wall and/or influenced the cardiohemodynamics of the small animal, which needs to be examined further. There may be a difference of ion channel distribution in the rat atria compared with human atria; namely, $\mathrm{I}_{\text {to }}$ contributes significantly to the repolarization phase of the action potential of rat atria unlike in human atria (3). Since pathogenesis of Af in the rat model will be different from those in humans, antiarrhythmic effects in this rat model may not necessarily reflect those in humans. As only 1 dose of one drug was assessed in this study, the pharmacological utility of this model needs to be determined by assessing multiple drugs in a wide range of doses.

In conclusion, the present Af model of rats may offer an alternative to current techniques. The example provided in this report demonstrates the potential widespread application of this new Af model of rats.

\section{Acknowledgments}

This study was supported in part by Grants-in-Aid from the Ministry of Education, Culture, Sports, Science, and Technology of Japan (\#15590222 and \#17590216) and Yamanashi Research Center of Clinical Pharmacology.

\section{References}

1 Friedrichs GS. Experimental models of atrial fibrillation/flutter. J Pharmacol Toxicol Methods. 2000;43:117-123.

2 Wakimoto H, Maguire CT, Kovoor P, Hammer PE, Gehrmann J, Triedman JK, et al. Induction of atrial tachycardia and fibrillation in the mouse heart. Cardiovasc Res. 2001;50:463-473.

3 Haugan K, Lam HR, Knudsen CB, Petersen JS. Atrial fibrillation in rats induced by rapid transesophageal atrial pacing during brief episodes of asphyxia: a new in vivo model. J Cardiovasc Pharmacol. 2004;44:125-135.

4 Iwasa A, Okumura K, Tabuchi T, Tsuchiya T, Tsunoda R, Matsunaga $\mathrm{T}$, et al. Effects of pilsicainide and propafenone on vagally induced atrial fibrillation: role of suppressant effect in conductivity. Eur J Pharmacol. 1998;356:31-40.

5 Kumagai K, Abe H, Hiraki T, Nakashima H, Oginosawa Y, Ikeda $\mathrm{H}$, et al. Single oral administration of pilsicainide versus infusion of disopyramide for termination of paroxysmal atrial fibrillation: a multicenter trial. Pacing Clin Electrophysiol. 2000;23:1880-1882.

6 Verheule S, Sato T, Everett IV T, Engle SK, Otten D, Rubartvon der Lohe $\mathrm{M}$, et al. Increased vulnerability to atrial fibrillation in transgenic mice with selective atrial fibrosis caused by overexpression of TGF- $\beta 1$. Circ Res. 2004;94:1458-1465.

7 Skolleborg KC, Gronbech JE, Grong K, Abyholm FE, Lekven J. Distribution of cardiac output during pentobarbital versus midazolam/fentanyl/fluanisone anesthesia in the rat. Laboratory Animals. 1990;24:221-227.

8 Griffioen KJS, Venkatesan P, Huang ZG, Wang X, Bouairi E, Evans C, et al. Fentanyl inhibits GABAergic neurotransmission to cardiac vagal neurons in the nucleus ambiguus. Brain Res. 2004;1007:109-115.

9 Lindmar R, Loffelholz K, Weide W. Inhibition by pentobarbital of the acetylcholine release from the postganglionic parasympathetic neuron of the heart. J Pharmacol Exp Ther. 1979;210:166-173.

10 Chiba S, Tsuboi M. Dominant anti-vagal effect of pentobarbital on cardiac responses to intracardiac autonomic nerve stimulation in the dog. Jpn J Pharmacol. 2001;86:248-250.

11 Nattel S, Wang Z, Matthews C. Direct electrophysiological actions of pentobarbital at concentrations achieved during general anesthesia. Am J Physiol. 1990;259:H1743-H1751.

12 Bachmann A, Mueller S, Kopp K, Brueggemann A, Suessbrich $\mathrm{H}$, Gerlach $\mathrm{U}$, et al. Inhibition of cardiac potassium currents by pentobarbital. Naunyn Schmiedebergs Arch Pharmacol. 2002; 365:29-37.

13 Gilat E, Rubinstein I, Binah O. Effect of sodium pentobarbital on the transmembrane action potential and the slow inward current of guinea pig ventricular myocytes. J Cardiovasc Pharmacol. 1987;10:485-488.

14 Lessa MA, Rodrigues E, Tibirica E. Cardioprotective action of fentanyl in a model of central sympathetic overactivity in rabbits: antiarrhythmic and anti-ischemic effects. Acta Anaesthesiol Scand. 2004;48:1115-1122.

15 Hunt GB, Ross DL. Comparison of effects of three anesthetic agents on induction of ventricular tachycardia in a canine model of myocardial infarction. Circulation. 1988;78:221-226. 